\title{
IL-16/miR-125a axis controls neutrophil recruitment in pristane-induced lung inflammation
}

Siobhan Smith, ${ }^{1}$ Pei Wen Wu, ${ }^{2,3}$ Jane J. Seo, ${ }^{2,3}$ Thilini Fernando, ${ }^{2,3}$ Mengyao Jin, ${ }^{2,3}$ Jorge Contreras, ${ }^{2,3}$ Erica N. Montano, ${ }^{2,3}$ Joan Ni Gabhann, ${ }^{1}$ Kyle Cunningham, ${ }^{2,3}$ Amro Widaa, ${ }^{4}$ Eoghan M. McCarthy, ${ }^{5}$ Eamonn S. Molloy, ${ }^{6}$ Grainne Kearns, ${ }^{5}$ Conor C. Murphy, ${ }^{1}$ Weiping Kong, ${ }^{7}$ Harry Björkbacka, ${ }^{8}$ Hardy Kornfeld, ${ }^{9}$ Lindsy Forbess, ${ }^{2}$ Swamy Venuturupalli, ${ }^{2}$ Mariko Ishimori, ${ }^{2}$ Daniel Wallace, ${ }^{2}$ Michael H. Weisman, ${ }^{2}$ and Caroline A. Jefferies ${ }^{2,3}$

'Department of Molecular and Cellular Therapeutics, Royal College of Surgeons in Ireland, Dublin, Ireland. 2Division of Rheumatology, Department of Medicine, Cedars-Sinai Medical Center, Los Angeles, California, USA. ${ }^{3}$ Department of Biomedical Sciences, Cedars-Sinai Medical Center, Los Angeles, California, USA. ${ }^{4}$ Department of Anatomy, Royal College of Surgeons in Ireland, Dublin, Ireland. ${ }^{5}$ Department of Rheumatology, Beaumont Hospital, Dublin, Ireland. ${ }^{6}$ Department of Rheumatology, St. Vincent's University Hospital, Dublin, Ireland. ${ }^{7}$ China-Japan Friendship Hospital, Chaoyang District, Beijing, China. ${ }^{8}$ Experimental Cardiovascular Research Unit, Department of Clinical Sciences, Lund University, Malmö, Sweden. ${ }^{9}$ Department of Medicine, University of Massachusetts Medical School, Worcester, Massachusetts, USA.

Severe lung inflammation and alveolar hemorrhage can be life-threatening in systemic lupus erythematosus (SLE) patients if not treated early and aggressively. Neutrophil influx is the driver key of this pathology, but little is known regarding the molecular events regulating this recruitment. Here, we uncover a role for IL-16/mir-125a in this pathology and show not only that IL-16 is a target for miR-125a but that reduced miR-125a expression in SLE patients associates with lung involvement. Furthermore, in the pristane model of acute "SLE-like" lung inflammation and alveolar hemorrhage, we observed reduced pulmonary miR-125a and enhanced IL-16 expression. Neutrophil infiltration was markedly reduced in the peritoneal lavage of pristane-treated IL-16deficient mice and elevated following i.n. delivery of IL-16. Moreover, a miR-125a mimic reduced pristane-induced IL-16 expression and neutrophil recruitment and rescued lung pathology. Mechanistically, IL-16 acts directly on the pulmonary epithelium and markedly enhances neutrophil chemoattractant expression both in vitro and in vivo, while the miR-125a mimic can prevent this. Our results reveal a role for miR-125a/IL-16 in regulating lung inflammation and suggest this axis may be a therapeutic target for management of acute lung injury in SLE.

Authorship note: SS and PWW contributed equally to this work.

Conflict of interest: The authors have declared that no conflict of interest exists.

Submitted: March 5, 2018 Accepted: June 26, 2018 Published: August 9, 2018

Reference information: JCI Insight. 2018;3(15):e120798. https://doi.org/10.1172/jci. insight.120798.

\section{Introduction}

Systemic lupus erythematosus (SLE) is an autoimmune disease with diverse clinical presentations that can affect multiple organs (1). Acute alveolar hemorrhage is a rare and potentially devastating form of acute respiratory distress syndrome $(2,3)$, with a mortality rate of $50 \%-70 \%$. As with many other forms of acute respiratory inflammation, including acute lung injury $(4,5)$, alveolar hemorrhage in SLE is associated with a massive influx of neutrophils into the airways $(6,7)$. Recently, neutrophils have been shown to be highly pathogenic in lupus cardiovascular and kidney disease, releasing inflammatory cytokines and immunostimulatory DNA in the form of neutrophil extracellular traps (NETs) (8-10). This subset of inflammatory, low-density neutrophils readily release NETs enriched in oxidized mitochondrial DNA, which further drives inflammation and type I IFN production. What drives inflammation and recruitment of neutrophils to the lung in SLE is currently not known.

Type I IFNs and particularly IFN- $\alpha$ have emerged as key pathogenic cytokines in SLE $(11,12)$. Elevated IFN- $\alpha$ is observed in over $50 \%$ of patients and correlates with disease severity, flare, and tissue involvement. In recent years, a type I IFN gene signature has been described in the peripheral blood of SLE patients that correlates with increased disease activity (13-15). In addition, genetic association 
studies have identified a number of genes that are linked to IFN production or signaling, such as IRF5, TYK2, IFIH1, and TLR7 $(16,17)$. Type I IFNs have also recently been found to be central to inflammatory responses in the lung in response to viral or environmental challenges. Type I IFNs can induce the expression of multiple chemokines, such as CCL2/MCP-1, CCL3/MIP-1 $\alpha$, CXCL1, CXCL2, and CXCL10/IP-10, influencing inflammation via recruitment and activation of multiple cell types, including neutrophils, monocytes, and $\mathrm{T}$ cells in the lung (18-27). While initially thought of largely as a $\mathrm{T}$ cell chemoattractant, CXCL10 has been shown to play an important role in lung inflammation via regulating neutrophil recruitment (28).

Supporting a role for type I IFNs regulating lung inflammation in SLE, pristane-inducible murine lupus results in severe lung inflammation and pulmonary hemorrhaging in $70 \%-80 \%$ of mice when pristane is administered on a C57BL/6 (B6) background (29, 30). TLR7-dependent production of type I IFN production drives the pathology of this model, with inflammatory type I IFN-producing monocytes detectable in the peritoneal cavity as early as 3 days after pristane injection. Importantly, this pristane-induced hemorrhage is lung specific and not due to a generalized change in vascular permeability (29). While IL-6 and IL-10 are reportedly increased in the bronchiolar lavage from pristane-treated mice, common inflammatory cytokines, such as TNF or IFN- $\gamma$, are not, suggesting that novel inflammatory cytokines or chemokines may be at play, driving neutrophil and inflammatory monocyte accumulation and associated pathology (29).

As with many human diseases, dysregulated expression of noncoding RNAs, including microRNA, has been associated with immunopathology of SLE (31). miR-125a is one such microRNA. Its expression is reportedly decreased in SLE patients and regulates a number of important immunoregulatory processes, including the expression of inflammatory cytokines and chemokines (32). Importantly, miR$125 \mathrm{a}$ is associated with a number of inflammatory diseases, including SLE (32), rheumatoid arthritis (33), and Crohn's disease (34). More recently, miR-125a has been shown to play a role in protecting against LPS-induced acute lung inflammation via its ability to regulate neutrophil development and infiltration into the lung in response to LPS (35).

Our studies demonstrate that not only are miR-125a levels decreased in SLE patient monocytes and peripheral blood mononuclear cells (PBMCs), but that decreased levels strongly associate with lung involvement. We identify IL-16, a proinflammatory cytokine known to drive airway inflammation (3642), as a novel and direct target of miR-125a. Importantly IL-16 levels are increased in SLE patients and associate with lung involvement. In the pristane model of IFN-driven pulmonary inflammation and hemorrhage, miR-125a levels are decreased in the lung as early as 3 days after pristane administration; this is accompanied by an increase in neutrophil recruitment and IL-16 expression, while i.n. administration of recombinant IL-16 exacerbates these effects. In vivo delivery of a miR-125a mimic on the other hand reduces IL-16 expression and protects against pristane-induced lung inflammation, with chemokines such as CCL2 and CXCL10 affected. Our results reveal that IL-16 acts directly on lung epithelial cells to activate p38 MAPK and NF- $\mathrm{KB}$ and subsequent chemokine expression. Thus, alterations in miR$125 \mathrm{a}$ levels in the lung result in enhanced expression of the proinflammatory cytokine IL-16 that, in turn, exacerbates lung inflammation by driving chemokine expression by lung epithelial cells, suggesting that a novel IL-16/miR-125a axis regulates autoimmune-induced lung inflammation.

\section{Results}

miR-125a is decreased in SLE patient monocytes and targets IL-16 gene expression. In an initial effort to identify differentially expressed miRNA in monocytes isolated from SLE patients, expression of $>800 \mathrm{miR}$ NAs in pooled samples derived from 5 SLE and 5 healthy control monocytes were investigated using the nCounter Human miRNA Assay (NanoString Technologies). 32 miRNAs were altered in patients with SLE as compared with normal controls: 14 were increased greater than 2-fold in SLE patient monocytes compared with controls and 18 were decreased, including miR-155, which was previously reported to be decreased in SLE patients (Supplemental Figure 1; supplemental material available online with this article; https://doi.org/10.1172/jci.insight.120798DS1). Among these, miR-125a-5p (miR-125a), of interest due to previous reports showing an association with SLE (32), was found to be decreased in a larger cohort of monocytes derived from 15 patients with SLE and 18 healthy controls (Figure 1A). KLF13, a transcription factor known to regulate the chemokine RANTES is a reported target of miR-125a (32); however, no significant difference in KLF13 expression was observed in monocytes from SLE patients 
compared with healthy controls (Supplemental Figure 2A), prompting us to explore additional targets. Comparing 3 miRNA target prediction programs, Target scan, PicTar, and miRDB, we identified IL-16 as a putative target of miR-125a and determined that the $3^{\prime}$-UTR of IL-16 predicted to pair with miR$125 \mathrm{a}$ is evolutionarily conserved (Figure 1B). In keeping with IL-16 as a putative target, SLE monocytes expressed higher levels of $I L-16$ compared with monocytes from healthy controls (Figure $1 \mathrm{C}$ ), and serum levels of IL-16 protein were also significantly elevated compared with controls (Figure 1D).

To establish miR-125a as regulator of IL-16 gene expression, transfection of monocytes from healthy controls with a miR-125a mimic significantly reduced $I L-16$ expression (Figure $1 \mathrm{E}$ ), whereas a miR-125a antagomir enhanced its expression (Figure $1 \mathrm{~F}$ ). To determine if the effects of miR-125a on $I L-16$ expression were direct, we cloned 2 regions of the $I L-163^{\prime} \mathrm{UTR}$ into a luciferase reporter construct — one containing the conserved putative miR-125a-binding site (site 1) and the other containing a region that contained no miR-125a binding site, the unrelated fragment control (site 2). Cotransfection of HEK293T cells with the two reporter constructs with either the miR-125a mimic or negative control demonstrated that transfection of HEK293T cells with miR-125a mimic significantly attenuated site 1 luciferase activity but failed to inhibit activity of the site 2 luciferase construct (Figure 1G), indicating that miR-125a directly targets the 3 UTR of IL-16. RNA pulldown of biotinylated miR-125a or a negative control, which was transfected into HeLa cells for 24 hours, demonstrated that the $I L-16$ transcript was enriched in the miR-125a pulldowns, compared with negative control (Figure $1 \mathrm{H}$ ). Taken together, our data demonstrate that IL-16 is to our knowledge both a novel and direct target of miR-125a.

miR-125a and IL-16 levels associate with SLE lung involvement and are altered in pristane-inducible lung inflammation in B6 mice. To determine the functional relevance of enhanced IL-16 expression in SLE, we looked at the potential clinical relevance of alterations in IL-16 expression in patient serum. This analysis showed that a higher serum IL-16 level was positively associated with autoantibodies against double-stranded DNA (dsDNA), a marker of more severe disease phenotype (Supplemental Figure 2B). Interestingly, while no significant change in IL-16 levels was observed in SLE patients with renal (Supplemental Figure 2C) or CNS involvement (Supplemental Figure 2D), elevated levels of IL-16 were observed in SLE patients with lung involvement (Figure 2A), while miR-125a levels were significantly decreased (Figure 2B). 16 of 42 patients were diagnosed with lung involvement using the criteria outlined in the Methods, in keeping with documented levels (43).

A diagnosis of lung involvement in lupus rarely indicates the need for a lung biopsy clinically. Thus, to address a possible association between IL-16 and miR-125a in lupus lung involvement, we turned to the pristane model of IFN-inducible lupus. As expected pristane led to a significant influx of neutrophil populations (Supplemental Figure 3A) and $\mathrm{Ly}_{6} \mathrm{C}^{\text {hi }}$ and $\mathrm{Ly} \mathrm{C}^{\text {int }}$ monocytes (Supplemental Figure 3B) into the peritoneum as compared with that in mice treated with PBS. This increase was accompanied by an influx of neutrophils and Ly6 $\mathrm{C}^{\text {int }}$ monocytes (Supplemental Figure 3C) in the lungs of mice 3, 7 , and 14 days after pristane treatment. Importantly, no increase in $\mathrm{CD}^{+} \mathrm{T}$ cells was observed, whereas $\mathrm{CD}^{+} \mathrm{T}$ cell numbers were significantly increased on day 14 (Supplemental Figure 3D). Consistent with published reports, alveolar hemorrhage was observed 10-14 days after pristane injection, with tissue sections from pristane-injected mice showing more extensive infiltration of immune cells into the lung and alveolar hemorrhage (Supplemental Figure 3E).

Regarding the effects of this model on miR-125a and IL-16 levels in the lungs, we observed significantly reduced expression of murine miR-125a from day 7 onward after pristane administration compared with that in PBS-treated mice (Figure 2C), with a corresponding increase in Il-16 expression observed after day 7 (Figure 2D). Intracellular flow cytometric analysis of immune cells positive for IL-16 revealed that monocytes in the peritoneal cavity (Figure $2 \mathrm{E}$ ) and $\mathrm{CD} 4^{+} \mathrm{T}$ cells in the lung (Figure 2F) stained for IL-16 following pristane challenge. Importantly, lung expression of neither Klf13 nor its target gene, Ccl5/Rantes, was altered by pristane administration, indicating that the effects of miR-125a are independent of Klf13 in this setting (Supplemental Figure 3F). In keeping with the enhanced gene expression, IL-16 staining was increased in lung sections from pristane-treated mice (Supplemental Figure 3G). Regarding what could potentially drive the expression of IL-16 in pristane-induced SLE, splenocytes isolated from wild-type B6 mice were treated with LPS, IFN- $\alpha$, murine antinuclear antibody (mANA) complexes, CpG, IFN- $\gamma$, and IL-23 for 24 hours. We observed that LPS, IFN- $\alpha$, and IL-23 strongly induced expression of IL-16, whereas responses to mANA complexes and CpG were induced 1.5- and 2-fold, respectively, and did not reach significance (Figure 2G). Analysis of IL-16 expression 
A

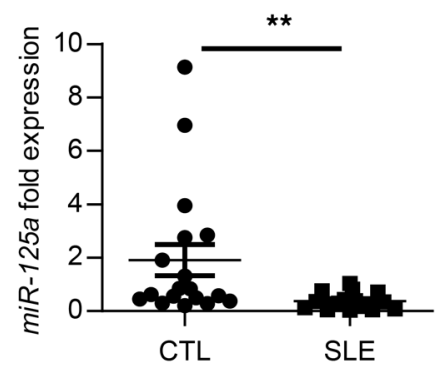

B

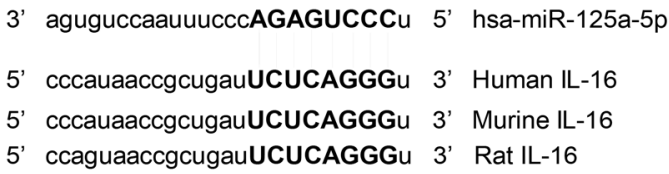

C

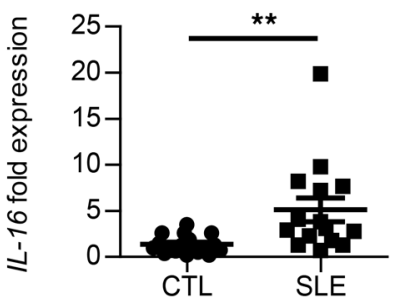

G

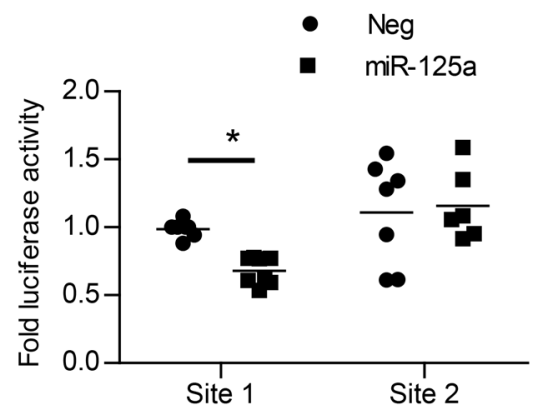

D

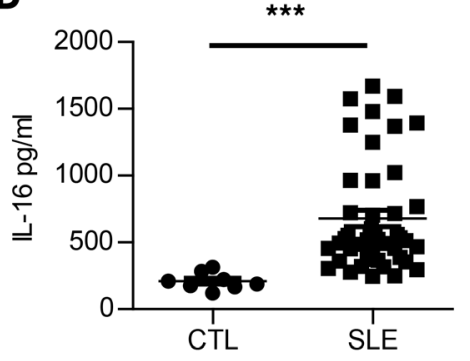

H

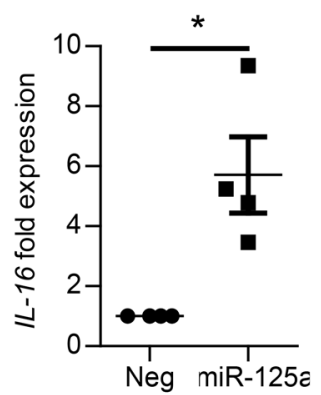

E

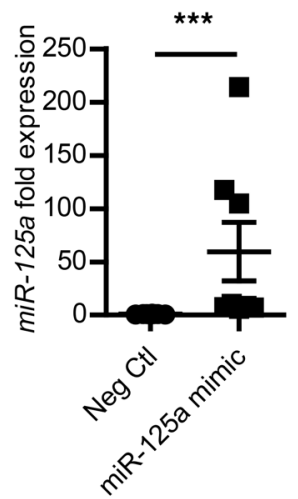

$\mathbf{F}$

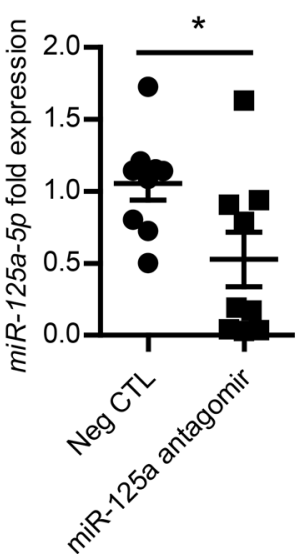

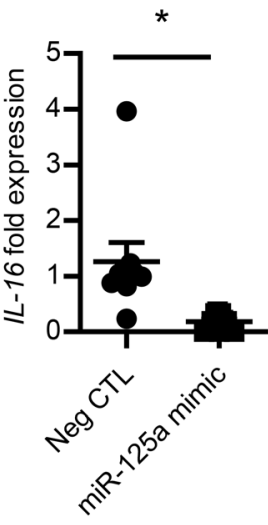

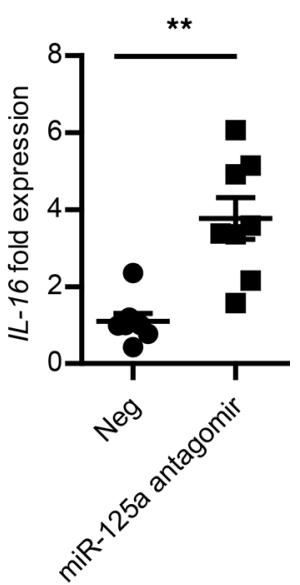

Figure 1. IL-16 a direct target for miR-125a in SLE monocytes. (A) hsa-miR-125a expression in 15 SLE patients and 18 healthy controls (CTL), as determined by qPCR. (B) Schematic representing conserved sequences of miR-125a-binding sites in the 3'-untranslated region (3' UTR) of IL-16 in human, rat, and mouse genomes. (C) Expression of IL-16 in 15 SLE patients and 16 healthy controls, as determined by qPCR. (D) Serum IL-16 in 42 SLE patients as compared with 10 healthy controls detected by ELISA. For A, C, and $\mathbf{D}$, each point represents a single subject; horizontal lines show the mean \pm SEM. ${ }^{* *} P \leq 0.01$, ${ }^{* * *} P \leq 0.001$. (E and F) Expression of miR-125a and IL-16 in miR-125a mimic- or anti-miR-302d-transfected monocytes, as determined by qPCR. (C) Luciferase activity in HEK293T cells transfected with the 3' UTR of IL-16 containing the miR-125a-binding site (site 1) and an unrelated fragment control (site 2) and either 50 nm negative control or miR-125a mimic. (H) Bi-miR-125a pulldown was performed followed by analysis of IL-16 expression by qPCR. For E-G, values represent the mean $\pm \mathrm{SD}$ of 3 samples; the data shown are representative of 3 independent experiments. ${ }^{*} P \leq 0.05,{ }^{*} P \leq 0.01$, as determined by Student's $t$ test.

in bone marrow-derived macrophages (BMDMs) from B6 or SLE-prone B6.SLE123 mice demonstrated that IFN- $\alpha$ could induce IL-16 expression in BMDMs from B6 mice, whereas enhanced expression of IL-16 was observed basally and following stimulation with both LPS and IFN- $\alpha$ of BMDMs from B6.SLE123 mice (Figure 2H).

In vivo delivery of a miR-125a mimic reduces IL-16 expression and neutrophil recruitment and attenuates pristane-induced lung inflammation. We next investigated whether in vivo manipulation of miR-125a levels could attenuate both IL-16 expression and lung inflammation by complexing 10 nmoles of a miR-125a mimic with Invivofectamine 3.0 and delivering it i.p. 1 day prior to pristane injection. Enhanced miR$125 a$ levels were observed in the lungs of miR-125a mimic-treated mice compared with those treated with negative control (Figure 3A). Analysis of lung digests between the different treatment groups (by qPCR and intracellular flow cytometry) showed that treatment with the miR-125a mimic significantly blocked 


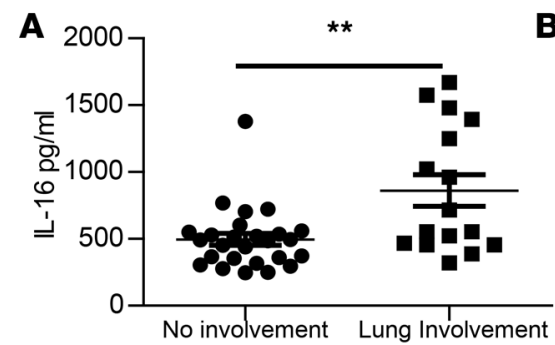

D

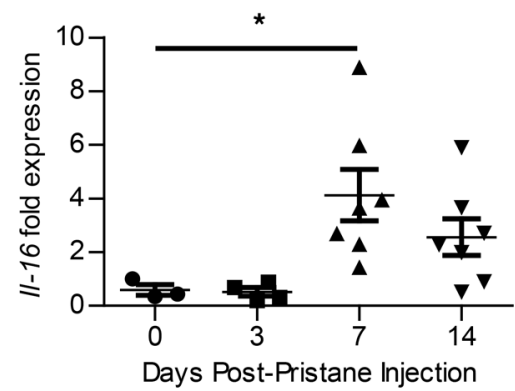

E
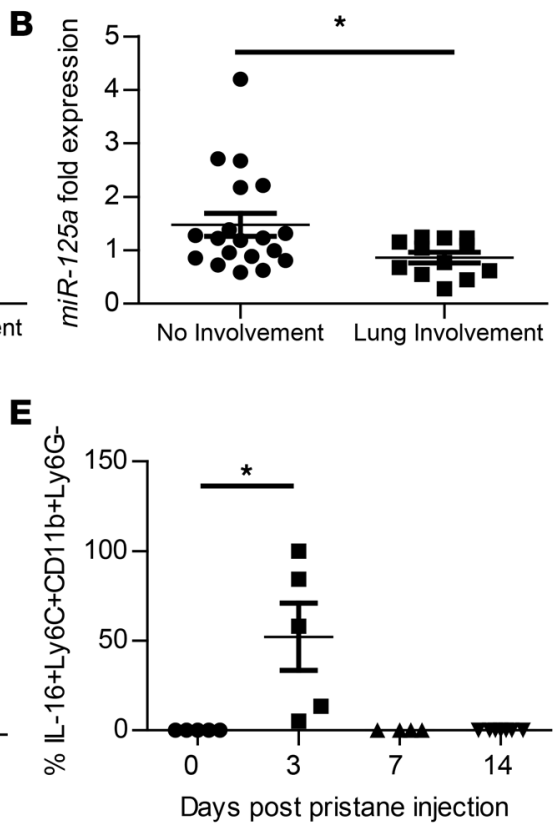

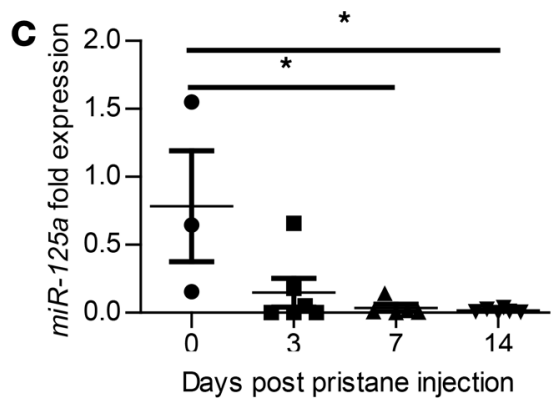

$\mathbf{F}$

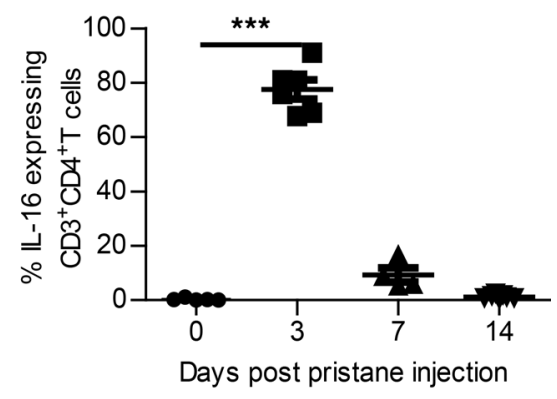

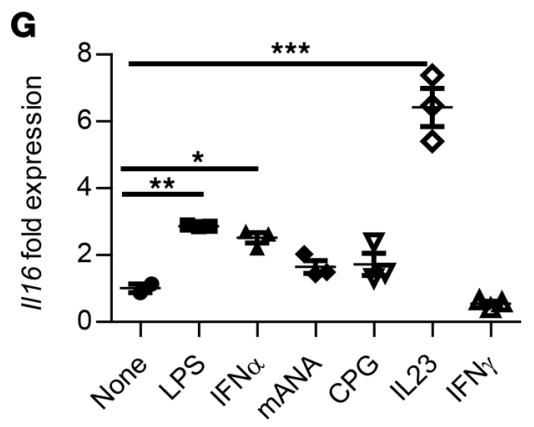

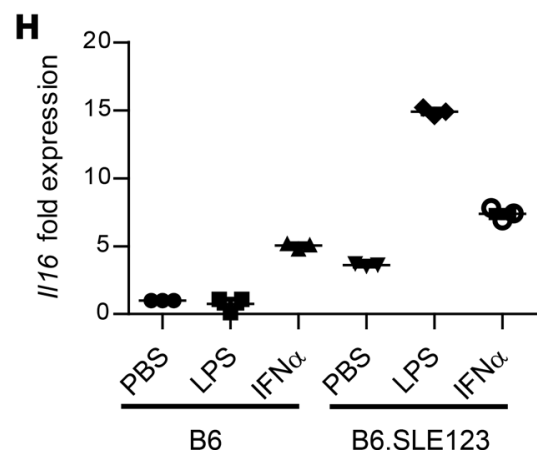

Figure 2. Altered levels of $\mathbf{m i R}-125 a$ and IL-16 associate with lung disease in SLE patients and in pristane-induced lung inflammation. (A) IL-16 serum levels in SLE patients with $(n=16)$ and without respiratory involvement $(n=24)$, as measured by ELISA. (B) miR-125a expression in SLE patients with ( $n=$ 12) and without respiratory involvement $(n=19)$, as determined by qPCR. (C and $\mathbf{D})$ Expression miR-125a (C) and $I I-16$ (D) was analyzed in the lungs of pristane-treated B6 mice by qPCR. (E and F) Percentage of IL-16-expressing (E) Ly6C ${ }^{\text {hi }}$ monocytes and (F) CD4+ $T$ cells shown following intracellular detection of IL-16 by flow cytometry. For C-F, $n=10$ for each group; data were analyzed using Mann Whitney $U$ test. (C) qPCR analysis of $I I-16$ expression in splenocytes isolated from wild-type B6 mice stimulated with LPS (1,000 ng/ml), IFN- $\alpha(500 \mathrm{U} / \mathrm{ml})$, IFN- $\gamma(500 \mathrm{U} / \mathrm{ml})$, murine antinuclear antibodies (ANA) (10 ng/ml), CpG $(3 \mu \mathrm{g} / \mathrm{ml})$, and IL-23 $(25 \mathrm{ng} / \mathrm{ml})$ for 3 hours. (H) Expression of $1 /-16$ in bone marrow-derived macrophages (BMDMs) isolated from wild-type B6 or SLEprone B6.SLE.123 mice stimulated with LPS $(100 \mathrm{ng} / \mathrm{ml})$ and IFN $-\alpha\left(10^{4} \mathrm{U} / \mathrm{ml}\right)$ for 6 hours. For $\mathbf{A}$ and $\mathbf{B}$, data shown represent mean $\pm \mathrm{SD}$. ${ }^{*} P \leq 0.05$, ${ }^{* *} P \leq$ 0.01 as determined by Mann Whitney $U$ comparison. For $\mathbf{C}-\mathbf{G}$, data shown represent mean \pm SD. ${ }^{*} P \leq 0.05,{ }^{* *} P \leq 0.01,{ }^{* *} P \leq 0.001$ as determined by 1 -way ANOVA. Values shown are from 2-3 independent experiments each with 3 replicates.

pristane-induced IL-16 expression at the mRNA (Figure 3B) and protein level (Figure 3C), in addition to reducing neutrophil influx (Figure 3D). Importantly, treatment with the miR-125a mimic appeared to target IL-16 produced by neutrophils to a greater extent than $\mathrm{CD}^{+} \mathrm{T}$ cells, as determined by intracellular flow cytometry of lung immune cells (Figure 3, E and F, respectively). H\&E staining of tissue sections from the lungs of miR-125a mimic-treated mice revealed less pristane-mediated alveolar hemorrhage compared with the negative control-treated group, as shown in Supplemental Figure 4A. It should be noted that, although the degree of alveolar hemorrhage observed was moderate rather than severe in the pristane group overall, mir-125a administration protected against lung damage in this model (Supplemental Figure 4B). Interestingly, miR-125a delivery did not alter recruitment of Ly6 $\mathrm{C}^{\mathrm{hi} / \mathrm{int}}$ monocytes, $\mathrm{CD}^{+} \mathrm{T}$ cells, or B cell numbers in the lung after pristane administration (Supplemental Figure 4, C-F). A reduction in the expression of 2 IFN-stimulated genes (ISGs), Isg15 and Mx1, was observed, although changes were not found to be significant (Figure 3, G and $\mathrm{H}$, respectively). 


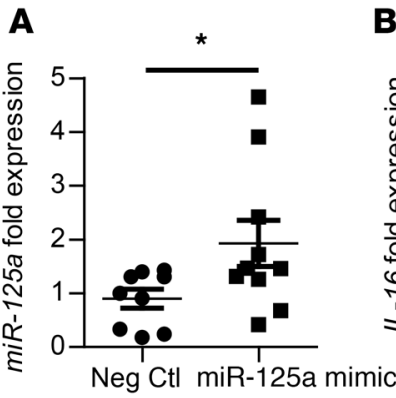

E

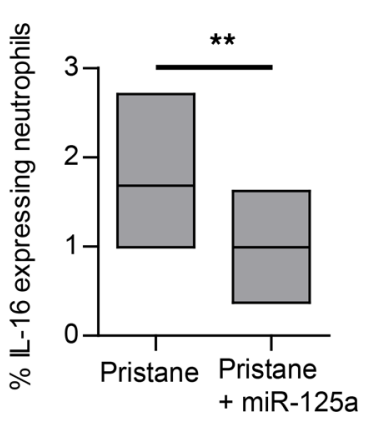

$\mathbf{F}$
B

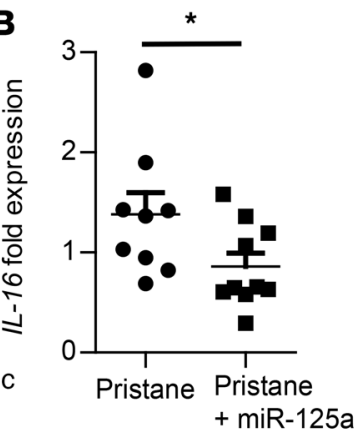

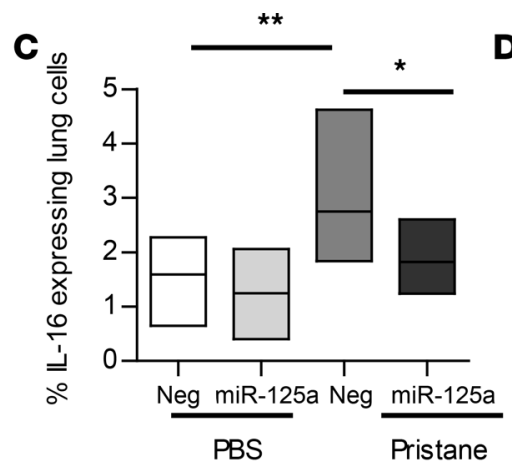
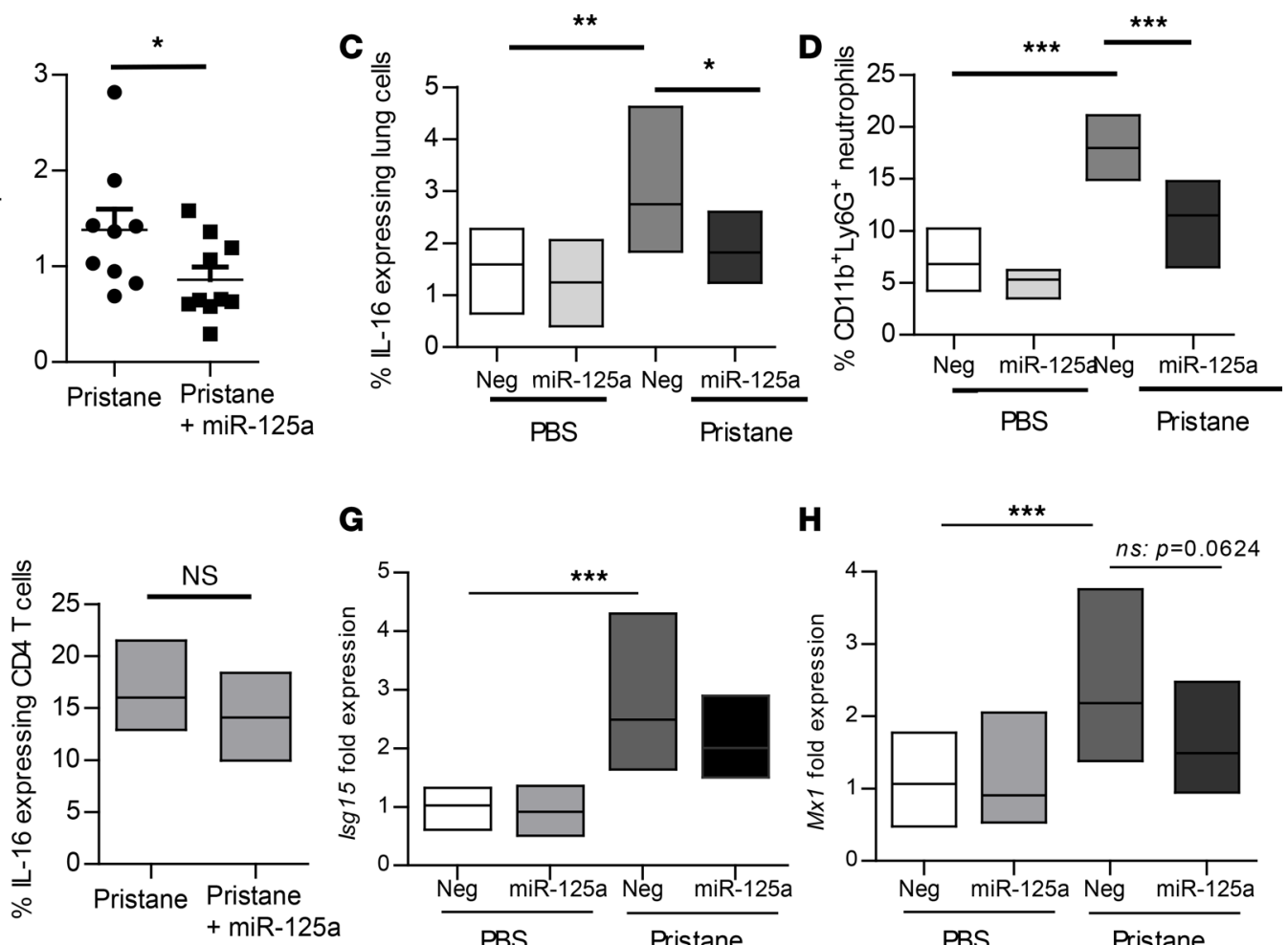

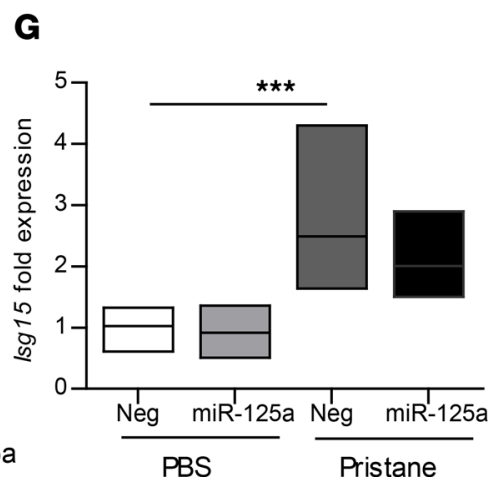

Figure 3. In vivo delivery of miR-125a mimic reduces neutrophil recruitment and IL-16 expression and attenuates pristane-induced lung pathology. (A-F) Mice received a single i.p. injection (10 nmol) of a miR-125a mimic or negative control ( $n=10$ for each treatment group) for 1 day before the i.p. injection of $500 \mathrm{ml}$ pristane followed by sacrifice at 10 days for analysis. (A) Expression of miR-125a is altered in the lungs following administration of miR-125a compared with negative control. (B) II-16 levels in the different treatment groups were analyzed in the lungs by qPCR. (C and D) Flow cytometry of cell populations in lung digests of the different treatment groups was performed. Percentage of IL-16-expressing cells (C) and neutrophils (D) in the lung. (E and F) Percentage of IL-16-expressing (E) Ly6G+ neutrophils and (F) CD4+ $4^{+}$cells following intracellular detection of IL-16 by flow cytometry. ( $\mathbf{G}$ and $\mathbf{H}$ ) Expression of $(\mathbf{G})$ Isg15 and $(\mathbf{H}) M x 1$ in the lungs of the different treatment groups analyzed by qPCR. For $\mathbf{A}, \mathbf{B}, \mathbf{E}$, and $\mathbf{F}$, data shown represent mean $\pm \mathrm{SD}$. ${ }^{*} P \leq 0.05$, ${ }^{*} P \leq 0.01$ as determined by unpaired $t$ test. For $\mathbf{C}, \mathbf{D}, \mathbf{G}$, and $\mathbf{H}$, data shown represent mean \pm SEM. ${ }^{*} P \leq$ $0.05,{ }^{* *} P \leq 0.01,{ }^{* *} P \leq 0.001$ as determined by 1 -way ANOVA.

IL-16 drives recruitment of neutrophils into the lung following pristane administration through induction of CXCL10 from lung epithelial cells. Considering the direct ability of IL-16 to induce lung inflammation, we assessed immune cell recruitment to the peritoneal cavity and lungs of pristane-treated IL-16-deficient mice. Our results showed that neutrophil recruitment alone was altered in the IL-16 $6^{-/}$mice in either compartment (Figure 4A). We next assessed the direct effect of recombinant IL-16 delivery on neutrophil recruitment. In the IL-16-deficient mice, intranasal (i.n.) delivery of IL-16 ( $1 \mu \mathrm{g} / \mathrm{mouse}, 24$ hours after administration of pristane and thereafter every 4 days) resulted in rescue of neutrophil recruitment in response to pristane (Figure 4A). In wild-type B6 mice, i.n. IL-16 alone had no effect in altering neutrophil recruitment (Figure 4B). However, in combination with pristane it was found to exacerbate recruitment of neutrophils (Figure 4B). With or without pristane, i.n. IL-16 failed to elicit changes in recruitment of inflammatory Ly6 $\mathrm{C}^{\text {hi }}$ monocytes or $\mathrm{CD} 4^{+} \mathrm{T}$ cells to the lung (Supplemental Figure 5, A and B, respectively). While i.n. IL-16 alone appeared to marginally (although not significantly) enhance expression of the ISGs Isg15 and MxI in the lung, no further enhancement of expression of either gene was observed following pristane injection (Supplemental Figure 5, C and D, respectively). Additionally, i.n. IL-16 further drove increased IL-16 production, which could be detected in the bronchoalveolar lavage fluid and systemically (Supplemental Figure 5, E and F, respectively). In assessing gene expression changes by qPCR in lung digests from pristane-treated mice, we observed a number of key chemokines that were elevated, including CXCL10, which was recently recognized as a key player in neutrophil-mediated lung inflammation (Supplemental Figure 5G) $(28,44)$. We therefore assessed the role of IL-16 in mediating CXCL10 levels and found that, while pristane alone could drive Cxcl10 expression in the lung, i.n. IL-16 significantly exacerbates pristane-driven $C x c l 10$ induction in the lungs 


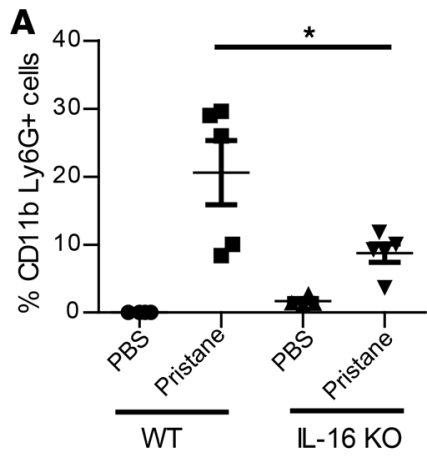

C

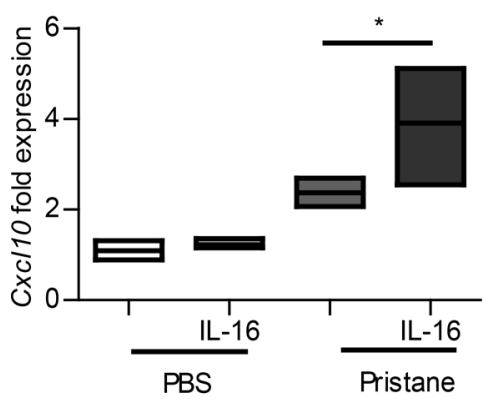

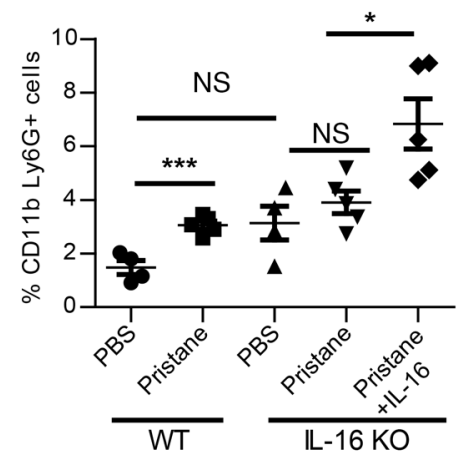

D

Effect of miR-125a in vivo on pristane-induced chemokine expression

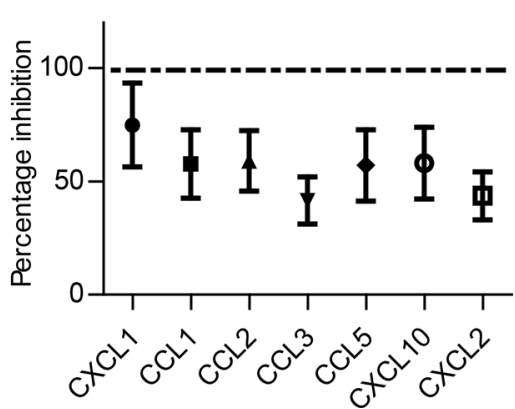

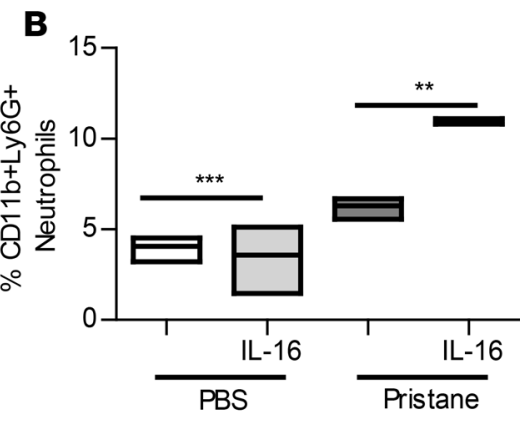

$\mathbf{E}$

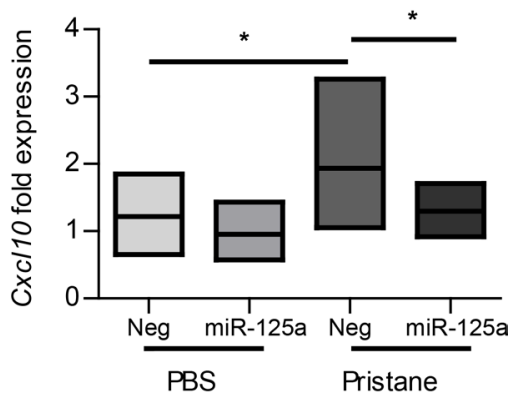

Figure 4. miR-125a/IL-16 axis drives pristane-induced cellular recruitment of neutrophils to the lung. (A) Flow cytometry analysis of neutrophils in the peritoneal lavage and lung digests of B6 or IL-16-/- mice following treatment with pristane or PBS $(n=8$ per group). (B) rmIL-16 ( $1 \mu \mathrm{g})$ was administered to B6 mice i.n. 1 day after pristane challenge followed by i.n. IL-16 every 4 days thereafter $(n=4-7$ for each group, 2 independent experiments). Flow cytometry analysis of the percentage of neutrophils in the lung digests of the different treatment groups at day 14 . (C) Cxc/10 expression in the lung in the different treatment groups, as measured by qPCR. (D) Mice received a single i.p. injection (10 nmol) of miR-125a mimic or negative control mimic $(n=10)$ for 1 day before pristane challenge followed by sacrifice at 10 days for analysis. miR-125a inhibited pristane-induced chemokine expression, as measured by qPCR. (E) Cxc/10 expression in the lungs in the different treatment groups, as measured by qPCR. Data were analyzed using the Mann Whitney $U$ test; mean $\pm \mathrm{SEM}$. ${ }^{*} P \leq 0.05,{ }^{* *} P \leq 0.01,{ }^{* * *} P \leq 0.001$.

of pristane-treated B6 mice (Figure 4C). Meanwhile in vivo delivery of a miR-125a mimic resulted in reduced chemokine expression, including Cxcl10 expression in the lungs in pristane-treated mice (Figure $4, \mathrm{D}$ and $\mathrm{E}$ ), further supporting a role for mir-125a as a negative regulator pristane-induced lung inflammation via its ability to target IL-16.

To assess whether IL-16 directly regulated chemokine expression in the lung, we analyzed Cxcl10 mRNA expression in murine precision cut lung slices (PCLSs) in response to IL-16 stimulation. As shown in Figure 5A, ex vivo stimulation of PCLSs with IL-16 induced an increase in Cxcl10 expression, whereas transfection of PCLS with miR-125a resulted in a reduction in both $\mathrm{Il}-16$ and $\mathrm{Cxcl10}$ expression (Figure 5B). Immunofluorescent detection of Cxc110 in PCLSs treated with IL-16 demonstrated that $\mathrm{Cxcl} 10$ protein levels were indeed elevated and that $\mathrm{Cxc110}$ appeared to colocalize with E-cadherin, a marker for epithelial cells (Figure 5C). As IL-16 has previously been shown to directly act on lung epithelial cells and induce apoptosis (36), we assessed the effects of IL-16 on the human bronchial epithelial cell line 16HBE14o-. IL-16 stimulation enhanced activity of p38 MAPK and NF-кB (32), as determined by Western blotting for phospho-p38 and phospho-p65 (Figure 5D), in addition to driving CXCL10 gene expression (Figure 5E). Inhibition of p38 MAPK, NF- $\mathrm{BB}$, or PKC pathways (previously reported to be activated by IL-16; ref. 45, 46) in either 16HBE14o- cells (Figure 5E) or murine PCLSs (Figure 5F) with SB203580 (p38 inhibitor), BMS-345541 (NF-кB inhibitor), and bisindolylmaleimide I (PKC inhibitor) blocked the ability of IL-16 to drive CXCL10 expression, indicating that IL-16 directly acts on lung epithelial cells to drive chemokine expression. Our studies, therefore, identify IL-16 as a target for mir-125a and demonstrate a pathogenic role for IL-16 in driving autoimmune lung inflammation via its ability to directly induce inflammation via direct effects on lung epithelial cells. 
A

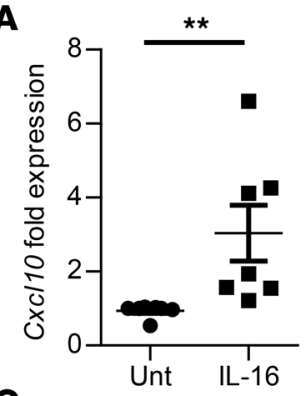

C

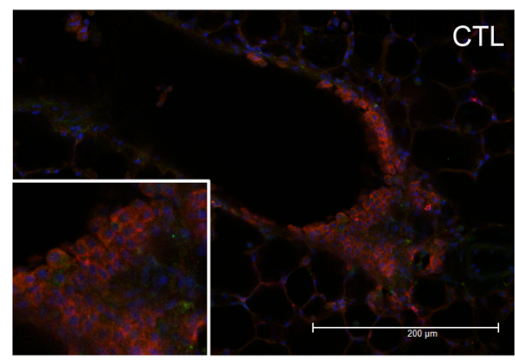

DAPI
B

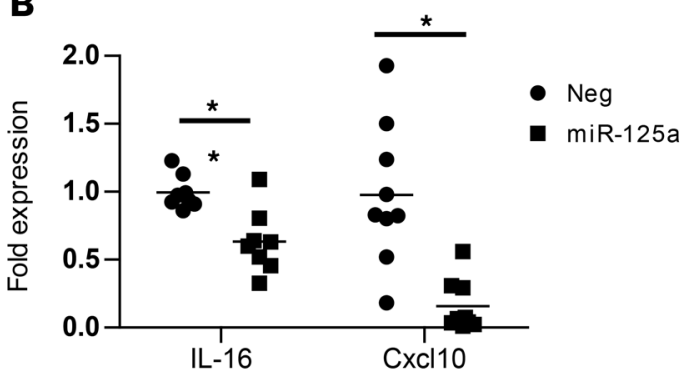

E

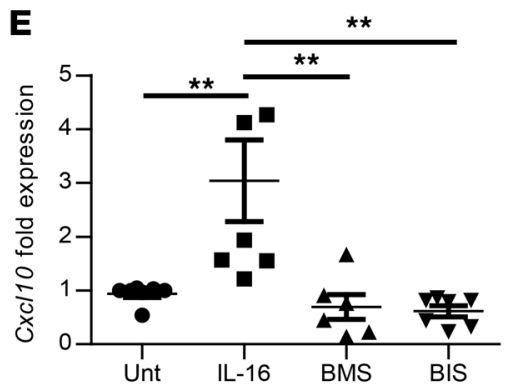

D

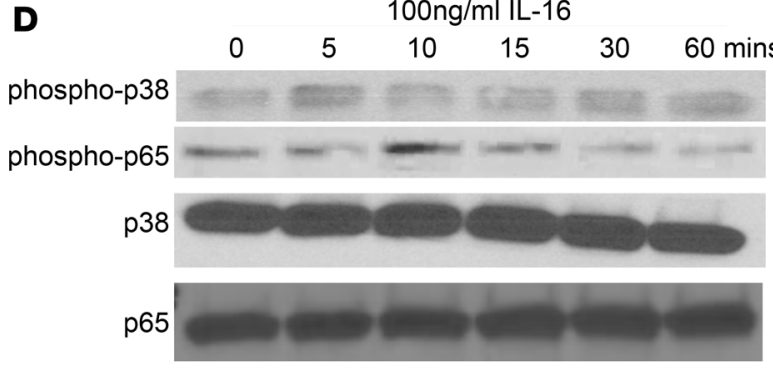

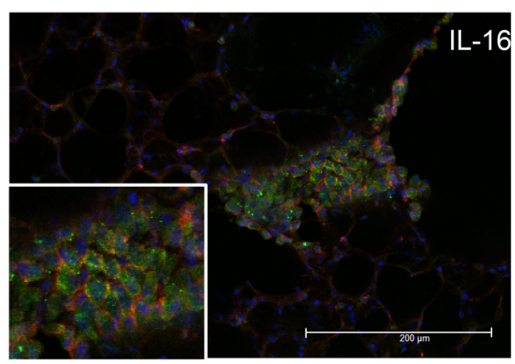

DAPI

E-Cadherin

Cxcl10

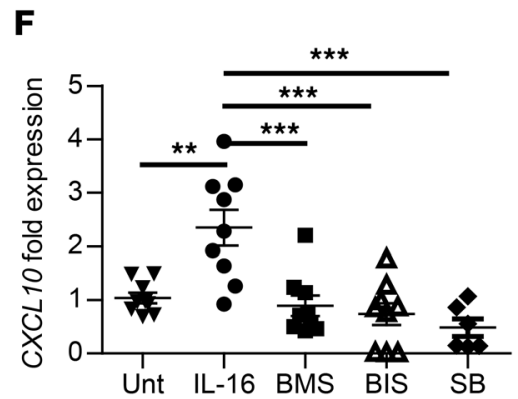

Figure 5. IL-16 acts directly on lung epithelial cells. (A and B) Precision cut lung slices (PCLS) were prepared from low-melting agarose-perfused wild-type B6 mouse lungs. Slices were (A) stimulated with IL-16 (100 ng/ml) for 6 hours or (B) transfected with a miR-125a mimic or negative control for 48 hours. Cxcl10 expression was analyzed by qPCR. A representative graph is shown; $n=3$. Mean $\pm \mathrm{SD}$. Data were analyzed using unpaired $t$ test. (C) PCLS were stimulated with IL-16 ( $50 \mathrm{ng} / \mathrm{ml}$ ) for 3 hours and immunostained for Cxc110 (green), E-cadherin (red), and DAPI (blue). Scale bar: $200 \mu \mathrm{m}$. Original magnification, $\times 20 ; \times 2$ (insets). (D) 16HBE140- cells were treated with IL-16 for different time points, as indicated. Expression of total p38, phospho-p38, total NF- $\kappa B$ p65, and phospho-NF- $\kappa B$ p65 was analyzed by immunoblotting. Activation of p38 and NF- $\mathrm{kB}$ was determined by the ratio of phosphorylated bands to total bands, respectively. Error bars represent SEM of 3 independently repeated experiments $(n=3)$. (E and $\mathbf{F}) 16$ HBE140- cells $(\mathbf{E})$ and PCLS $(\mathbf{F})$ were pretreated with inhibitors as indicated for 1 hour and stimulated with IL-16 for 6 hours followed by qPCR for CXCL10. For D-F, data were analyzed by 1-way ANOVA. ${ }^{*} P \leq 0.05,{ }^{* *} P \leq 0.01,{ }^{* * *} P \leq 0.001$.

\section{Discussion}

Acute autoimmune lung manifestations include alveolar hemorrhage - a devastating and potentially fatal complication associated with SLE as well as other autoimmune conditions. The immune events that mediate the pathology underlying this condition are relatively unknown, and very little information exits regarding what may influence predisposition to either lung involvement or acute autoimmune lung injury in SLE. In this study, we have identified a mechanism regulating lung inflammation in the context of SLE - decreased expression of the microRNA miR-125a associates with lung involvement via its ability to regulate the proinflammatory cytokine IL-16 directly. Mechanistically, we show that enhanced IL-16 expression in the murine lung drives enhanced recruitment of neutrophils to the lung via a direct ability to activate lung epithelial cells.

Type I IFNs are central to inflammatory responses in the lung, not only activating lung epithelial cells and resident immune cells, but also inducing the expression of cytokines and chemokines (CCL2/MCP-1, CCL3/MIP-1 $\alpha$, and CXCL10/IP-10, for example) and hence influencing inflammation via recruitment and activation of neutrophils, monocytes, and T cells in the lung (18-27). Our results show that IFN- $\alpha$ can induce IL-16 expression in murine splenocytes and BMDMs directly and that in the pristane model of SLE, 
an IFN- $\alpha$-driven model, IL-16 expression is induced both in the lung and systemically. IL-16 is a multifunctional inflammatory cytokine that acts on $\mathrm{T}$ cells, monocytes, and eosinophils, driving migration and regulating activity (36-42). Initially studied in the context of asthma, IL-16 was shown to recruit CCR5-expressing Th1 cells to sites of allergic inflammation and drive T regulatory cell expansion, acting to protect in models of Th2-driven asthma (47-50). However, in an inflammatory setting, IL-16 has been found to exacerbate Staphylococcus aureus infections in the lung; although, how it mediates its effects in this setting has not yet been fully elucidated (51). In models of chronic lung inflammation, such as bleomycin-induced lung injury, IL-16 is upregulated but does not appear to play a role in pathology in this model (52).

As to what regulates IL-16 expression in this model, we have shown that miR-125 directly regulates IL-16 expression in monocytes and that its expression is decreased in monocytes from SLE patients, in keeping with previous reports showing that miR-125a levels are decreased in PBMCs of SLE patients (32). In keeping with this observation, we report decreased miR-125a in SLE monocytes and identify the proinflammatory cytokine IL-16 as a possible novel and direct target of miR-125a, whose expression is elevated in SLE monocytes. Both gain- and loss-of-function studies demonstrate that transfecting monocytes with miR-125a mimic or antagomir has reciprocal effects on IL-16 expression, while analysis of the ability of biotinylated miR-125a to bind the 3' UTR of IL-16 confirms IL-16 as a direct target. Increased circulating IL-16 levels have been reported previously in SLE patients, with increased levels correlating positively with SLE disease activity scores (53). In keeping with, this we demonstrate an association between circulating IL-16 levels and dsDNA positivity status in our SLE cohort, a marker of more severe disease (54). More importantly, we observe a strong association between alterations in miR-125a and IL-16 levels in SLE patients who experienced respiratory involvement (namely pleuritis or alveolar hemorrhage) compared with those with other organ involvement, indicating a possible involvement of miR-125a-IL-16 in lung inflammation in the context of SLE. It is possible that the decreases in miR-125a and increases in IL-16 that we observe more generally across our SLE cohort reflect a phenomenon that has widely been reported - that autoimmune lung involvement, be it alveolitis, interstitial lung disease, or the observance of ground glass opacities, is more widely present but remains undetected in the absence of conducting pulmonary function tests (PFTs) or high-resolution CT (HRCT) scans. Such testing in rheumatoid arthritis patients, for example, with early-stage disease, has revealed a much higher level of lung involvement than previously thought, with $35 \%$ percent showing ground glass opacities on HRCT (55). In keeping with these reports, a number of studies have shown that asymptomatic SLE patients have a much higher involvement of lung inflammation in SLE on PFT or HRCT scan than previously stated $(56,57)$. Therefore, the possibility exists that unconfirmed subclinical lung involvement accounts for our observations.

Using the pristane model of acute autoimmune lung disease, we show that reduced miR-125a expression in the lungs of pristane-treated mice correlates with increased IL-16 expression and that CD $4^{+} \mathrm{T}$ cells and monocytes are the most likely source of IL-16 in this setting. However, while neutrophil numbers are increased in the pristane lung, surprisingly alterations in $\mathrm{CD}^{+} \mathrm{T}$ cells are not observed. A number of publications have shown that IL-16 can induce chemotaxis of other immune cells, such as monocytes and eosinophils, in addition to CD4 T cells $(40,41)$. However, in this model, it appears that IL-16 regulates neutrophil rather than $\mathrm{CD}^{+} \mathrm{T}$ cell recruitment, with i.n. administration of IL-16 enhancing pristane-induced neutrophil recruitment, but with no effect on $\mathrm{CD}^{+} \mathrm{T}$ cell recruitment. In contrast, administration of a miR-125a mimic completely reversed IL-16 expression in response to pristane and, more importantly, pristane-induced neutrophil accumulation. Importantly, these observed increases in neutrophil infiltration in the lung were associated with increased expression of chemokines, such as CXCL10, a chemokine known to regulate $\mathrm{CD}^{+} \mathrm{T}$ cell recruitment that has been shown to play an important role in lung pathology via its ability to regulate neutrophil recruitment (28).

In models of acute lung injury, the accumulation of neutrophils in the lung is CXCL10 dependent, driven in part by increased expression of CXCR3 on infiltrating neutrophils (28). Although we were not able to observe changes in CXCR3 expression in our model, we found that IL-16 acts directly on lung epithelial cells to enhance expression of CXCL10. The ability of IL-16 to directly induce chemokine expression from lung epithelial cells is supported by previous observations showing, first, that IL-16 directly acts on alveolar basal lung epithelial cells (36) and, second, that lung epithelial cells produce CXCL10 both in vitro and in vivo in response to human rhinovirus type 16 infection in an NF- $\mathrm{KB}-$ dependent manner (58). Our results show that IL-16 acts on bronchial epithelial cells to activate p38 MAPK, PKC, and NF- $\mathrm{B}$-dependent pathways $(45,46)$ to enhance expression of CXCL10. Our data therefore indicate that IL-16 can directly 
act on lung epithelial cells to induce upregulation of neutrophil-attracting chemokines, such as CXCL10. Importantly, in vivo delivery of miR-125a suppressed both IL-16 and CXCL10 expression and reduced neutrophil accumulation in the lungs of pristane-treated mice, supporting a key role for the miR-125/IL-16 axis in mediating neutrophil accumulation in the lung in the context of autoimmune lung inflammation. How neutrophils contribute to autoimmune lung pathology in this model remains to be elucidated. Recent work has demonstrated the role of low-density neutrophils and NETosis in SLE organ pathogenesis via the ability of low-density neutrophils to release potential autoantigens such as self-DNA and nuclear-associated material, specifically in the context of cardiovascular disease (8-10). Histologically, autoimmune diffuse alveolar hemorrhage of the lung is accompanied by a massive neutrophil influx that is thought to contribute to pathology through the release of reactive oxygen species and antimicrobial peptides, such as defensins (7). However, a number of studies have suggested that there is no role for neutrophil NETosis in the pathogenesis of lupus using MRL.Fas ${ }^{\mathrm{lpr}}$ mice crossed to either Pad4- or Nox2-deficient animals. Loss of Nox2 and NADPH oxidase activity resulted in an exacerbation of kidney disease (59), whereas PAD4 deficiency had no effect on glomerulonephritis in the MRL.Fas ${ }^{\text {pr }}$ model (60) but exacerbated both kidney disease and ANA production in pristane-induced lupus (61). Thus, further work is required to elucidate the role of neutrophils in end-organ disease and their contribution to autoimmune lung specifically.

Taken together, our results demonstrate an important association between miR-125a and its target IL-16 in SLE-associated lung involvement and reveal a role for IL-16 in acting directly on lung epithelial cells to drive chemokine expression and, hence, contribute to neutrophil accumulation. Our results reveal that modulation of IL-16 expression, either by direct i.n. administration or via targeting it with miR-125a mimics, alters neutrophil recruitment and, therefore, lung inflammation in the context of autoimmune lung inflammation. Our data strongly support a role for IL-16-induced chemokine expression in driving of neutrophil influx and potentially pathology and suggest the miR-125a/IL-16 axis as a target for autoimmune lung injury.

\section{Methods}

Patients and controls and handling of samples. All SLE patients were recruited from Beaumont Hospital; St. James's Hospital, Dublin, Ireland; St. Vincent's University Hospital; and Cedars-Sinai Medical Center, as per ACR diagnostic criteria (62). Clinical data were determined for each patient at the time of the blood draw (Table 1). Lung involvement was defined in this study as evidence of pleuritic chest pain, with evidence of a pleural rub, effusion, or thickening (SLEDAI-2K) (63) or pleurisy, pleural effusion with dyspnea, pulmonary hemorrhage, interstitial alveolitis/pneumonitis, and shrinking lung syndrome (BILAG 2004 index) (64). Age- and sex-matched healthy donors who had no history of autoimmune diseases or treatment with immunosuppressive agents were included.

Isolation of CD14+ monocyte cells. PBMCs were separated from the whole blood of patients with SLE and healthy control subjects by density-gradient centrifugation with Ficoll-Paque Plus (GE Healthcare). CD14 ${ }^{+}$ monocytes were purified from fresh PBMCs by positive selection using magnetic CD $14^{+}$beads (Miltenyi Biotec) according to the manufacturer's protocol. Purified monocytes were cultured in phenol red-free RPMI 1640 medium supplemented with $10 \%$ charcoal-stripped fetal calf serum and $100 \mu \mathrm{g} / \mathrm{ml}$ penicillin/ streptomycin.

Real-time PCR analysis. RNA was extracted from cell cultures using TRIzol reagent (MilliporeSigma) according to the manufacturer's protocol. Expression of $>800$ miRNAs was assessed by NanoString Technologies using nCounter miRNA Expression Assays. To validate the results, miR-125a expression was analyzed using the miScript primer assay (Qiagen, MS00003423) and miScript SYBR Green PCR kit (Qiagen), with normalization to the U6 small nuclear RNA. RNA was reverse transcribed using the iScript Reverse Transcription Supermix Kit (Bio-Rad) according to the manufacturer's recommendations. Real-time qPCR investigating human $I L-16, K L F 13$, and $C X C L 10$ gene expression was performed using appropriate primers (Table 2) with the SYBR Green Taq ReadyMix (MilliporeSigma) according to the manufacturer's recommendations. Data were analyzed using an ABI Prism 7900 system (Applied Biosystems) and were normalized to an 18S rRNA reference. Murine Il-16, Klf13, Mx1, Isg15, Ccl5, and Cxcl10 gene expression was performed using appropriate primers (Table 2) with the PerfecTa SYBR Green PCR Kit (Quanta, 95072-012) as per the manufacturer's recommendations. Data were analyzed in triplicate using an ABI Prism 7900HT system (Applied Biosystems) and were normalized to an L32 RNA reference. Real-time PCR data were analyzed using the $2^{-\mathrm{ddCt}}$ method (65). 
Table 1. Demographic characteristics of SLE patients involved in this study $(n=42)$

\begin{tabular}{|c|c|}
\hline \multicolumn{2}{|l|}{ Sex } \\
\hline Female & 37 \\
\hline Male & 5 \\
\hline Age, mean $( \pm \mathrm{SD})$ & $38.9( \pm 12.9)$ \\
\hline Disease duration, mean ( \pm SD) & $6.9( \pm 5.9$ \\
\hline \multicolumn{2}{|l|}{ ACR classification criteria } \\
\hline ANA & $42(100 \%)$ \\
\hline Malar rash & $30(71 \%)$ \\
\hline Discoid rash & $2(7 \%)$ \\
\hline Photosensitivity & $22(52 \%)$ \\
\hline Oral ulcers & $14(33 \%)$ \\
\hline Arthritis & $23(55 \%)$ \\
\hline Serositis & $18(45 \%)$ \\
\hline Renal & $14(33 \%)$ \\
\hline CNS disease & $8(19 \%)$ \\
\hline Hematologic & $31(73 \%)$ \\
\hline Immunologic disorder & 29 (69\%) \\
\hline SLEDAI (median \pm IQR) & $2(0-4)$ \\
\hline SLICC damage index (median \pm IQR) & $0(0-1)$ \\
\hline Lung involvement & $16 / 42$ \\
\hline \multicolumn{2}{|l|}{ Autoantibody profile } \\
\hline Anti-dsDNA & $29(69 \%)$ \\
\hline Anti-Ro & $16(38 \%)$ \\
\hline Anti-La 7 & $(16 \%)$ \\
\hline Anti-RNP & $6(14 \%)$ \\
\hline Anti-Sm & $4(10 \%)$ \\
\hline \multicolumn{2}{|l|}{ Current immunosuppression } \\
\hline Prednisolone & $20(47 \%)$ \\
\hline Hydroxychloroquine & $33(78 \%)$ \\
\hline Azathioprine & $13(30 \%)$ \\
\hline Mycophenolate mofetil & $4(10 \%)$ \\
\hline Cyclophosphamide & $1(2 \%)$ \\
\hline
\end{tabular}
mM Tris- $\mathrm{HCl}, \mathrm{pH} 6.8,10 \%$ SDS, $0.5 \%$ bromophenol blue, $50 \%$ glycerol, $50 \mathrm{nM}$ DTT) and boiled at $95^{\circ} \mathrm{C}$ for 10 minutes. Equal quantities of whole-cell lysates were resolved by electrophoresis on a denaturing SDS-polyacrylamide gel according to the method of Laemmli (66) and transferred to a nitrocellulose membrane for immunoblotting. The antibodies used for immunoblotting included anti- $\alpha$-actinin (Cell Signaling Technology), anti-phosphorylated p38 MAPK (Cell Signaling Technology), anti-p38 MAPK (Cell Signaling Technology), anti-phosphorylated p65 (Santa Cruz Biotechnology), and anti-p65 (Santa Cruz Technology). Following immunoblotting, the membrane was developed using enhanced chemiluminescent HRP substrate (Millipore). Optical densitometry was performed using Image Studio Lite (LI-COR Bioscience).

Mimic and inhibitor transfection. MicroRNA-125a oligonucleotides were obtained from Dharmacon. Negative controls were based on the sequences of miRNA in Caenorhabditis elegans (cel-miR-67). Reverse transfection of human primary monocytes was performed using Metafectene SI transfection reagent (Biontex Laboratories $\mathrm{Gmbh}$ ) as recommended by the manufacturer's protocol. Evaluation of the experiment was carried out 48-72 hours after transfection.

Luciferase reporter assay. The putative miR-125a target sequence in the $3^{\prime}$ UTR of human IL-16 (site 1) and an unrelated fragment control region of the $3^{\prime}$ UTR of human IL-16 (site 2) was cloned into the psiCHECK-2 vector (Promega) downstream of the Renilla luciferase reporter gene with the following primers: site 1, forward, 5'-GAGAGACTCGAGCTGAAGCCAAAGCCAATA-3' and reverse, 5'-GAGAGAGCGGCCGCTGTCATATGAAATTCTAATA-3'; site 2, forward, 5'-GAGAGACTCGAGGCTTAATGATAATATTGTGG-3' and reverse, 5'-GAGAGAGCGGCCGCTATGAGTGAATGATCTCACG-3'.

All constructs were sequenced and were prepared with the use of an EndoFree Plasmid Maxi kit (Qiagen). 293T cells were seeded at $1 \times 10^{5}$ cells/ well in a 96-well plate 1 day before transfection and then transfected with a mixture of 50 ng IL-16-UTR site 1 or site 2 luciferase reporter vector and 50 $\mathrm{nM}$ of either a negative control or miR-125a mimic. The cells were harvested 24 hours later, and luciferase activity was assessed using a Dual Luciferase Reporter Assay System (Promega). Firefly luciferase was used to normalize the Renilla luciferase. All experiments were carried out in triplicate.

Biotin miR-125a pulldown. HeLa cells $\left(1 \times 10^{6}\right.$ cells/well $)$ were transfected in triplicate with Bi-miR-125a or Bi-negative control miRNA (Dharmacon) as above for 24 hours. Briefly, cells were lysed in $1 \mathrm{ml}$ lysis buffer (20 mM Tris [pH 7.5], $100 \mathrm{mM} \mathrm{KCL}, 5 \mathrm{mM} \mathrm{MgCl}, 0.3 \%$ NP-40, 50 U RNase OUT [Invitrogen], and complete mini-protease inhibitor cocktail [Roche]) and incubated on ice for 5 minutes. Streptavidin-coated magnetic beads (Invitrogen) were blocked for 2 hours at $4^{\circ} \mathrm{C}$ in lysis buffer with $1 \mathrm{mg} / \mathrm{ml}$ yeast tRNA and $1 \mathrm{mg} / \mathrm{ml}$ BSA (Ambion), followed by washing with lysis buffer. Cytoplasmic lysate was added to beads and incubated for 4 hours at $4^{\circ} \mathrm{C}$ before washing 5 times with $1 \mathrm{ml}$ lysis buffer. RNA bound to the beads was isolated using Trizol. IL-16 mRNA levels in the Bi-miR-125a or Bi-negative control pulldown were quantified by qPCR with normalization to the housekeeping gene $G A P$ $D H$. An enrichment ratio of pulldown RNA to input levels was calculated for IL-16.

Mice. B6 mice were purchased from The Jackson Laboratory. Mice were propagated and maintained in the animal facility at the Department of Comparative Medicine (Davis Building) of Cedars-Sinai Medical Center. IL-16 ${ }^{-/-}$mice were generated by Sue Kim (University of Massachusetts Medical School) and HK (67) and supplied by HB. Animals were between 6 and 12 weeks old at the time of experimentation. Lung cellular infiltration was induced by i.p. administration of $0.5 \mathrm{ml}$ pristane (MilliporeSigma). Lung tissues were collected for RNA extraction and embedded in paraffin for sectioning. The miR-125a and negative control mimics were purchased from Dharmacon. Invivofectamine 3.0 in vivo transfection reagent was purchased from Thermo Fisher Scientific. The transfection protocol was based on the manufacturer's instructions. Briefly, $100 \mu \mathrm{l}$ of $1.2 \mathrm{mg} / \mathrm{ml}$ miRNA solution was prepared by adding $50 \mu 1$ complexation 
Table 2. Human and murine primer sequences used in qPCR

\begin{tabular}{|c|c|c|}
\hline Human KLF13 & CGGCCTCAGACAAAGGGTC & TTCCCGTAAACTTTCTCGCAG \\
\hline Murine IL-16 & AAGAGCCGGAAATCCACGAAA & GTGCGAGGTCTGGGATATTGC \\
\hline Murine KLF13 & CCTCAGACAAAGGGGTCGG & GTAGTGGCACTTGTCCTTCC \\
\hline Murine CXCL10 & CCAGTAGTGAGAAAGGGTCG & AGGGCTTGGGGCAAATTGTT \\
\hline Murine MX1 & GATCCGACTTCACTTCCACATCG & СATCTCAGTGGTAGTCAACCC \\
\hline
\end{tabular}

buffer to $50 \mu \mathrm{l}$ of $2.4 \mathrm{mg} / \mathrm{ml}$ solution of miR-125a or negative control. This solution was immediately added to $100 \mu 1$ Invivofectamine 3.0 transfection reagent, which was then mixed well to ensure complete complexation. The mixture was incubated at $50^{\circ} \mathrm{C}$ for 30 minutes. The complex was then diluted 6 -fold with sterile PBS, pH 7.4, and mixed well. For each injection, we used $200 \mu 1$ of working solution (10 nm of the miR-125a mimic/negative control mimic).

To assess direct effects of IL-16 on the lung, IL-16 was i.n. delivered 1 day after pristane challenge and then followed by i.n. delivery every 4 days until day 14. Mice were sacrificed for tissue and blood 14 days after injection unless otherwise indicated. Recombinant IL-16 was purchased from Cell Guidance Systems (catalog GFM49) and had $\leq 1.00 \mathrm{EU} / \mu \mathrm{g}$ endotoxin, as measured by LAL.

Immunohistochemistry. The mice were sacrificed, and lungs were perfused with ice-cold PBS prior to removal. Lungs were fixed for 24 hours in 10\% neutral buffered formalin then stored in $70 \%$ ethanol until they were embedded in paraffin and sectioned at $5 \mu \mathrm{m}$. The slices were deparaffinized in xylene and rehydrated to graded changes of ethanol. Antigen retrieval was performed using a pressure cooker by heating the sections for 45 minutes at $95^{\circ} \mathrm{C}$ in sodium citrate buffer, $\mathrm{pH}$ 6.0. IL-16 staining was performed using the Anti-Rabbit HRP-DAPI Cell \& Tissue Staining kit as per the manufacturer's protocol (R\&D Systems, CTS005). Briefly, endogenous peroxidase was exhausted with peroxidase blocking reagent for 5 minutes followed by incubation with rabbit serum-blocking reagent for 15 minutes to minimize nonspecific staining. Sections were then incubated with avidin-blocking reagent for 15 minutes followed by biotin blocking reagent for a further 15 minutes. The sections were incubated overnight at $4^{\circ} \mathrm{C}$ with a rabbit primary antibody against IL-16 (dilution 1:200; Santa Cruz, sc-7902) followed by HRP-conjugated secondary antibody for 30 minutes. The sections were then incubated with HSS-HRP followed by several washes of PBS. The immunohistochemical reaction was visualized by incubation with 3,3'-diaminobenzidine tetrahydrochloride chromagen and sections were counterstained with hematoxylin. Images were captured using Aperio ScanScope AT Turbo.

Flow cytometry. Single-cell suspensions from lungs and peritoneal lavage were isolated from wildtype naive and pristane-injected B6 mice. Briefly, $10^{6}$ cells were suspended in FACS buffer contain-

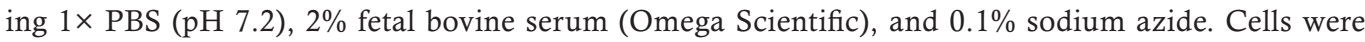
stained in the relevant fluorochrome-labeled mAbs, and 10,000 events were acquired on a BD Biosciences LSRII. For IL-16 intracellular staining, cell fixation and permeabilization using Cytofix/ Cytoperm buffer (BD Biosciences) were performed before antibody staining. Data analysis was performed by using FlowJo V10 (Tree Star). The following monoclonal antibodies (Biolegend) were used: CD11b-APC, clone M1/70; Ly6G-FITC, clone 1A8; Ly6C-PerCP, clone HK1.4; CD3-APC, clone 17A2; CD4-FITC, clone RM4-5; CD8-PE, clone 53-6.7; CD19-Pacific Blue, clone 6D5; and IL-16-PE, clone 14.1. Fluorochrome- and isotype-matching mAbs were used as negative controls.

PCLS preparation and culture. Wild-type B6 mice were sacrificed by isoflurane inhalation, and lungs were perfused with $15 \mathrm{ml}$ PBS at room temperature through the right ventricle. The trachea was cannulated with a 20-gauge catheter (20 G $\times 1.00$ in. BD Insyte Autoguard), and then lung lobes were inflated with $3 \%$ $(\mathrm{w} / \mathrm{v}) 45^{\circ} \mathrm{C}$ prewarmed low-melting agarose (Promega). The inflated lung lobes were immediately removed and cooled on ice for 15 minutes. After lobes solidified, the left lobe was separated and sectioned into 
$200-\mu \mathrm{m}$-thick slices using a vibratome (Leica VT 1200S, Leica Microsystems). Tissue slices were then cultured in DMEM supplemented with $10 \% \mathrm{FCS}, 100 \mu \mathrm{g} / \mathrm{ml}$ penicillin/streptomycin, and glutamine. Inhibitors and stimulants were applied immediately after sectioning.

Macrophage culture. Isolation and activation of BMDMs were performed as previously described (68).

Cell culture. 16HBE140- bronchial epithelial cells (ATCC) were seeded in 6-well plates at a density of $5 \times 10^{5}$ cells/well in MEM with Earle's salts, supplemented with $10 \%$ FBS and incubated at $37^{\circ} \mathrm{C}$ in $5 \% \mathrm{CO}_{2}$ until confluence ( $2-3 \mathrm{~d}$ ). Cells were stimulated with recombinant human IL-16 (Origene, catalog TP720036; endotoxin levels $<1.00 \mathrm{EU} / \mu \mathrm{g}$, as measured by LAL) for the indicated time points prior to lysis and analysis of gene expression or protein phosphorylation.

Statistics. Data were analyzed using Prism 4 software, version 4.03 (GraphPad Software). Statistical analysis was performed with the 2-tailed Student's $t$ test or Mann-Whitney $U$ test when the data were not normally distributed. Multiple comparisons between groups were performed using 1-way ANOVA, followed by post hoc analysis using Tukey's comparison. Statistical significance was defined as a $P<0.05$.

Study approval. All SLE patients were recruited from Beaumont Hospital, St. James's Hospital, and St. Vincent's University Hospital. All participants provided informed written consent, and the study received prior approval from the institutional ethics review boards at Beaumont Hospital, St. James's Hospital, and St. Vincent's University Hospital. The use of human PBMCs and monocytes from patients and controls was reviewed and approved by the ethics review board at the Royal College of Surgeons in Ireland and the IRB of Cedars-Sinai Medical Center.

\section{Author contributions}

SS, PWW, JJS, and CAJ designed the experiments, acquired data, and prepared the manuscript. TF, MJ, WK, JNG, AW, CCM, KC, ENM, and JC aided in data acquisition. EMM, ESM, GK, LF, SV, MI, DW, and $\mathrm{MHW}$ provided clinical samples. $\mathrm{HB}$ and $\mathrm{HK}$ provided reagents for this study

\section{Acknowledgments}

We would like to thank D. Howard, R.Z. Lee, and P. O'Connell (Department of Rheumatology, Beaumont Hospital); G. Cunnane and M.F. Doran (Department of Rheumatology, St. James's Hospital); and R. Conway and P. Gallagher (Department of Rheumatology, St. Vincent's University Hospital) for their involvement in patient recruitment. Support for this study was provided in part by National Institutes of Health grant HL0127384 (to HK), Cedars-Sinai Medical Center (to CAJ), the Lupus Research Alliance (ALR/TIL/332436) (to CAJ), the Arthritis Foundation (AF2017-433570), and the Irish Research Council (GOIPD/2014/512) (to CAJ), which had no role in study design, data collection and analysis, decision to publish, or preparation of the manuscript. We would like to acknowledge Sue Kim for her work in making the IL-16 ${ }^{-/}$mice.

Address correspondence to: Caroline Jefferies, Division of Rheumatology, Department of Medicine and Department of Biomedical Sciences, Cedars-Sinai Medical Center, 8700 Beverly Blvd., Los Angeles, California 90048, USA. Phone: 310.423.8658; E-mail: Caroline.Jefferies@cshs.org.

1. Bengtsson AA, Rönnblom L. Systemic lupus erythematosus: still a challenge for physicians. J Intern Med. 2017;281(1):52-64.

2. Kamen DL, Strange C. Pulmonary manifestations of systemic lupus erythematosus. Clin Chest Med. 2010;31(3):479-488.

3. Agmon-Levin N, Selmi C. The autoimmune side of heart and lung diseases. Clin Rev Allergy Immunol. 2013;44(1):1-5.

4. Grommes J, Soehnlein O. Contribution of neutrophils to acute lung injury. Mol Med. 2011;17(3-4):293-307.

5. Abraham E. Neutrophils and acute lung injury. Crit Care Med. 2003;31(4 Suppl):S195-S199.

6. Cordier JF, Cottin V. Alveolar hemorrhage in vasculitis: primary and secondary. Semin Respir Crit Care Med. 2011;32(3):310-321.

7. Zamora MR, Warner ML, Tuder R, Schwarz MI. Diffuse alveolar hemorrhage and systemic lupus erythematosus. Clinical presentation, histology, survival, and outcome. Medicine (Baltimore). 1997;76(3):192-202.

8. Carmona-Rivera C, Zhao W, Yalavarthi S, Kaplan MJ. Neutrophil extracellular traps induce endothelial dysfunction in systemic lupus erythematosus through the activation of matrix metalloproteinase-2. Ann Rheum Dis. 2015;74(7):1417-1424.

9. Knight JS, Kaplan MJ. Lupus neutrophils: 'NET' gain in understanding lupus pathogenesis. Curr Opin Rheumatol. 2012;24(5):441-450.

10. Garcia-Romo GS, et al. Netting neutrophils are major inducers of type I IFN production in pediatric systemic lupus erythematosus. Sci Transl Med. 2011;3(73):73ra20.

11. Crow MK. Type I interferon in systemic lupus erythematosus. Curr Top Microbiol Immunol. 2007;316:359-386.

12. Rönnblom L. The importance of the type I interferon system in autoimmunity. Clin Exp Rheumatol. 2016;34(4 Suppl 98):21-24 
13. Kennedy WP, et al. Association of the interferon signature metric with serological disease manifestations but not global activity scores in multiple cohorts of patients with SLE. Lupus Sci Med. 2015;2(1):e000080.

14. Petri M, et al. Longitudinal expression of type I interferon responsive genes in systemic lupus erythematosus. Lupus. 2009;18(11):980-989.

15. Bauer JW, et al. Interferon-regulated chemokines as biomarkers of systemic lupus erythematosus disease activity: a validation study. Arthritis Rheum. 2009;60(10):3098-3107.

16. Bronson PG, Chaivorapol C, Ortmann W, Behrens TW, Graham RR. The genetics of type I interferon in systemic lupus erythematosus. Curr Opin Immunol. 2012;24(5):530-537.

17. Byrne JC, et al. Genetics of SLE: functional relevance for monocytes/macrophages in disease. Clin Dev Immunol. 2012;2012:582352.

18. Goritzka M, et al. Alveolar macrophage-derived type I interferons orchestrate innate immunity to RSV through recruitment of antiviral monocytes. J Exp Med. 2015;212(5):699-714.

19. Lin KL, Suzuki Y, Nakano H, Ramsburg E, Gunn MD. CCR2+ monocyte-derived dendritic cells and exudate macrophages produce influenza-induced pulmonary immune pathology and mortality. J Immunol. 2008;180(4):2562-2572.

20. Shahangian A, et al. Type I IFNs mediate development of postinfluenza bacterial pneumonia in mice. J Clin Invest. 2009;119(7):1910-1920.

21. Weighardt $\mathrm{H}$, et al. Type I IFN modulates host defense and late hyperinflammation in septic peritonitis. J Immunol. 2006;177(8):5623-5630.

22. Lehmann MH, Torres-Domínguez LE, Price PJ, Brandmüller C, Kirschning CJ, Sutter G. CCL2 expression is mediated by type I IFN receptor and recruits NK and T cells to the lung during MVA infection. J Leukoc Biol. 2016;99(6):1057-1064.

23. Rot A, von Andrian UH. Chemokines in innate and adaptive host defense: basic chemokinese grammar for immune cells. Annu Rev Immunol. 2004;22:891-928.

24. Charo IF, Ransohoff RM. The many roles of chemokines and chemokine receptors in inflammation. N Engl J Med. 2006;354(6):610-621.

25. Johnston B, Burns AR, Suematsu M, Issekutz TB, Woodman RC, Kubes P. Chronic inflammation upregulates chemokine receptors and induces neutrophil migration to monocyte chemoattractant protein-1. J Clin Invest. 1999;103(9):1269-1276.

26. Lee SC, et al. Cutaneous injection of human subjects with macrophage inflammatory protein-1 alpha induces significant recruitment of neutrophils and monocytes. J Immunol. 2000;164(6):3392-3401.

27. Ramos CD, et al. MIP-1alpha[CCL3] acting on the CCR1 receptor mediates neutrophil migration in immune inflammation via sequential release of TNF-alpha and LTB4. J Leukoc Biol. 2005;78(1):167-177.

28. Ichikawa A, et al. CXCL10-CXCR3 enhances the development of neutrophil-mediated fulminant lung injury of viral and nonviral origin. Am J Respir Crit Care Med. 2013;187(1):65-77.

29. Chowdhary VR, Grande JP, Luthra HS, David CS. Characterization of haemorrhagic pulmonary capillaritis: another manifestation of Pristane-induced lupus. Rheumatology (Oxford). 2007;46(9):1405-1410.

30. Reeves WH, Lee PY, Weinstein JS, Satoh M, Lu L. Induction of autoimmunity by pristane and other naturally occurring hydrocarbons. Trends Immunol. 2009;30(9):455-464.

31. Zan H, Tat C, Casali P. MicroRNAs in lupus. Autoimmunity. 2014;47(4):272-285.

32. Zhao X, et al. MicroRNA-125a contributes to elevated inflammatory chemokine RANTES levels via targeting KLF13 in systemic lupus erythematosus. Arthritis Rheum. 2010;62(11):3425-3435.

33. Murata K, et al. Comprehensive microRNA analysis identifies miR-24 and miR-125a-5p as plasma biomarkers for rheumatoid arthritis. PLoS ONE. 2013;8(7):e69118

34. Pan W, et al. MiR-125a targets effector programs to stabilize Treg-mediated immune homeostasis. Nat Commun. $2015 ; 6: 7096$.

35. Qin Y, et al. MiR-125a Is a critical modulator for neutrophil development. PLoS Genet. 2017;13(10):e1007027.

36. Yadav S, Shi Y, Wang H. IL-16 effects on A549 lung epithelial cells: dependence on CD9 as an IL-16 receptor? J Immunotoxicol. 2010;7(3):183-193.

37. Cruikshank WW, et al. IL-16 inhibition of CD3-dependent lymphocyte activation and proliferation. J Immunol. 1996;157(12):5240-5248.

38. Theodore AC, Center DM, Nicoll J, Fine G, Kornfeld H, Cruikshank WW. CD4 ligand IL-16 inhibits the mixed lymphocyte reaction. J Immunol. 1996;157(5):1958-1964

39. Center DM, Kornfeld H, Cruikshank WW. Interleukin-16. Int J Biochem Cell Biol. 1997;29(11):1231-1234.

40. Cruikshank WW, Berman JS, Theodore AC, Bernardo J, Center DM. Lymphokine activation of T4+ T lymphocytes and monocytes. J Immunol. 1987;138(11):3817-3823.

41. Rand TH, Cruikshank WW, Center DM, Weller PF. CD4-mediated stimulation of human eosinophils: lymphocyte chemoattractant factor and other CD4-binding ligands elicit eosinophil migration. J Exp Med. 1991;173(6):1521-1528.

42. Cruikshank WW, et al. Molecular and functional analysis of a lymphocyte chemoattractant factor: association of biologic function with CD4 expression. Proc Natl Acad Sci USA. 1994;91(11):5109-5113.

43. Todd NW, Wise RA. Respiratory complications in the collagen vascular diseases. Clin Pulm Med. 1996;3(2):101-112.

44. Michalec L, et al. CCL7 and CXCL10 orchestrate oxidative stress-induced neutrophilic lung inflammation. J Immunol. 2002;168(2):846-852.

45. Krautwald S. IL-16 activates the SAPK signaling pathway in CD4+ macrophages. J Immunol. 1998;160(12):5874-5879.

46. Parada NA, Cruikshank WW, Danis HL, Ryan TC, Center DM. IL-16- and other CD4 ligand-induced migration is dependent upon protein kinase C. Cell Immunol. 1996;168(1):100-106.

47. Pinsonneault S, El Bassam S, Mazer B, Cruikshank WW, Laberge S. IL-16 inhibits IL-5 production by antigen-stimulated T cells in atopic subjects. J Allergy Clin Immunol. 2001;107(3):477-482.

48. McFadden C, et al. Preferential migration of T regulatory cells induced by IL-16. J Immunol. 2007;179(10):6439-6445.

49. de Bie JJ, Henricks PA, Cruikshank WW, Hofman G, Nijkamp FP, van Oosterhout AJ. Effect of interleukin-16-blocking peptide on parameters of allergic asthma in a murine model. Eur J Pharmacol. 1999;383(2):189-196.

50. De Bie JJ, et al. Exogenous interleukin-16 inhibits antigen-induced airway hyper-reactivity, eosinophilia and Th2-type cytokine 
production in mice. Clin Exp Allergy. 2002;32(11):1651-1658.

51. Ahn DS, Parker D, Planet PJ, Nieto PA, Bueno SM, Prince A. Secretion of IL-16 through TNFR1 and calpain-caspase signaling contributes to MRSA pneumonia. Mucosal Immunol. 2014;7(6):1366-1374.

52. Glass WG, et al. Generation of bleomycin-induced lung fibrosis is independent of IL-16. Cytokine. 2009;46(1):17-23.

53. Lee S, Kaneko H, Sekigawa I, Tokano Y, Takasaki Y, Hashimoto H. Circulating interleukin-16 in systemic lupus erythematosus. Br J Rheumatol. 1998;37(12):1334-1337.

54. ter Borg EJ, Horst G, Hummel EJ, Limburg PC, Kallenberg CG. Measurement of increases in anti-double-stranded DNA antibody levels as a predictor of disease exacerbation in systemic lupus erythematosus. A long-term, prospective study. Arthritis Rheum. 1990;33(5):634-643.

55. Metafratzi ZM, et al. Pulmonary involvement in patients with early rheumatoid arthritis. Scand J Rheumatol. 2007;36(5):338-344.

56. Pego-Reigosa JM, Medeiros DA, Isenberg DA. Respiratory manifestations of systemic lupus erythematosus: old and new concepts. Best Pract Res Clin Rheumatol. 2009;23(4):469-480.

57. Quadrelli SA, et al. Pulmonary involvement of systemic lupus erythematosus: analysis of 90 necropsies. Lupus. 2009;18(12):1053-1060

58. Spurrell JC, Wiehler S, Zaheer RS, Sanders SP, Proud D. Human airway epithelial cells produce IP-10 (CXCL10) in vitro and in vivo upon rhinovirus infection. Am J Physiol Lung Cell Mol Physiol. 2005;289(1):L85-L95.

59. Campbell AM, Kashgarian M, Shlomchik MJ. NADPH oxidase inhibits the pathogenesis of systemic lupus erythematosus. Sci Transl Med. 2012;4(157):157ra141.

60. Gordon RA, et al. Lupus and proliferative nephritis are PAD4 independent in murine models. JCI Insight. 2017;2(10):e92926.

61. Kienhöfer D, et al. Experimental lupus is aggravated in mouse strains with impaired induction of neutrophil extracellular traps. JCI Insight. 2017;2(10):e92920.

62. Tan EM, et al. The 1982 revised criteria for the classification of systemic lupus erythematosus. Arthritis Rheum. 1982;25(11):1271-1277.

63. Gladman DD, Ibañez D, Urowitz MB. Systemic lupus erythematosus disease activity index 2000. JRheumatol. 2002;29(2):288-291.

64. Yee CS, et al. British Isles Lupus Assessment Group 2004 index is valid for assessment of disease activity in systemic lupus erythematosus. Arthritis Rheum. 2007;56(12):4113-4119.

65. Livak KJ, Schmittgen TD. Analysis of relative gene expression data using real-time quantitative PCR and the 2(-Delta Delta C(T)) Method. Methods. 2001;25(4):402-408

66. Laemmli UK. Cleavage of structural proteins during the assembly of the head of bacteriophage T4. Nature. 1970;227(5259):680-685.

67. Ren F, et al. Pro-IL-16 regulation in activated murine CD4+ lymphocytes. J Immunol. 2005;174(5):2738-2745.

68. Ní Gabhann J, et al. Btk regulates macrophage polarization in response to lipopolysaccharide. PLoS One. 2014;9(1):e85834. 